\title{
Osteopathie bei einem Hund mit Bewegungsstörung - ein Fallbericht
}

Anne Lisa Louis

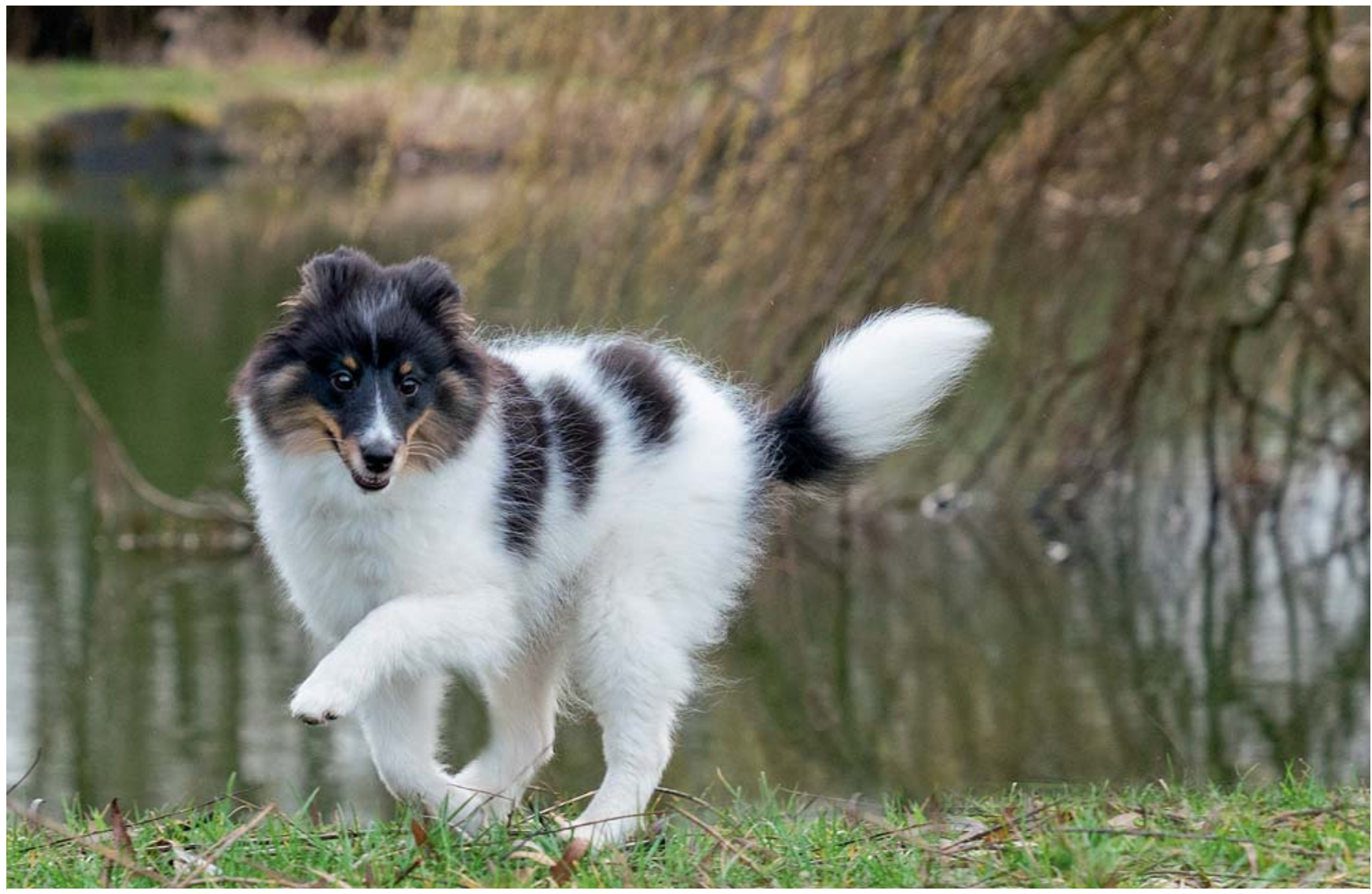

Vom „Hoppeln“ zu Bergtouren und Spaziergängen durch Wald und Flur - durch die osteopathische Behandlung wurde neue Bewegung möglich und Balance wiederhergestellt (Symbolbild). Quelle: Kirsten Oborny, Thieme Gruppe

Im Folgenden stelle ich einen Fall aus meiner osteopathischen Praxis vor, der sicherlich einer der herausragendsten für mich bleiben wird. Einerseits, weil ein so schöner Erfolg zu sehen war und immer noch ist, und andererseits, weil ich selbst viel in den Behandlungen lernen durfte. Dieser Hund lehrte mich, mich mit meinen Vorstellungen und Ideen zurückzuhalten und einfach mit dem zu arbeiten, was der Patient anbietet oder zulässt. Ich wurde damit belohnt, sehen zu dürfen, wie neue Bewegung möglich wird und eine Balance entsteht - und bestehen bleiben kann.

\section{Anamnese}

Es handelt sich um die Border Collie Hündin Bella. Bei Erstvorstellung im August 2017 ist sie 14 Monate alt. Sie war bereits einmal, ohne weitere Auffälligkeiten, läufig.
Der Grund für die Konsultation ist eine Bewegungsstörung, die laut Besitzerin seit etwa dem 6. Lebensmonat sichtbar ist.

In schnelleren Gangarten als Schritt kann Bella hinten nur hoppeln und ihre Hinterfüße nicht einzeln setzen. Dabei 
ist es egal, ob sie in der Ebene oder am Berg geht. Auch beim Treppensteigen kann sie die Stufen nur hoppelnd überwinden.

Die Besitzerin hat Bella deswegen bereits sehr umfangreich bei Kollegen untersuchen lassen. Röntgenologisch gibt es an Wirbelsäule, Hüfte, Knien, Tarsalgelenken sowie den Ellbogen keine auffälligen Befunde.

Bella ist ein sehr agiler, flinker Hund, der außerhalb des Hauses nicht zur Ruhe kommen kann und auf alle Reize blitzschnell reagiert. Stillhalten, -sitzen oder -liegen ist nicht so ihre Sache. Zuhause kann sie das, aber in ablenkungsreicher Umgebung reicht ihre Konzentration dafür nicht aus. Aber sie kann inzwischen glücklicherweise auch in stressigen Situationen fressen.

Bisher hat die Besitzerin versucht, Bella durch Übungen für die Koordination zu einem verbesserten Körpergefühl zu verhelfen. Sie tut dies mit viel Fachwissen und Umsicht. Sie bearbeitet auch intensiv die Problemzonen des Alltags mit ihrer Hündin (Umweltunsicherheit, Stressbewältigung, rassetypische Geräuschempfindlichkeit, Impulskontrolle).

\section{Erstvorstellung (08.08.2017)}

\section{Adspektion und Ganganalyse}

Zusätzlich zu den bereits berichteten Befunden fällt adspektorisch beim Besuch in der Praxis auf, dass Bella so stark kuhhessig steht, dass die Fersenhöcker sich berühren. Sie zieht die Lendenwirbelsäule (LWS) eher auf und stellt die Hintergliedmaßen unter. Die Ellbogen sind mittelgradig eingedreht.

Bellas Gangbild zeigen laut Besitzerin auch die Geschwister ihres Hundes.

In der freien Bewegung beim Erkunden der Praxis stellt Bella das Becken sehr steil, sobald sie die Nase Richtung Boden senkt. Die Wirbelsäule verändert dabei ihre Position nicht nennenswert, die LWS kann sie also nicht gut in Flexion bringen.

Bella lässt sich von Fremden nicht so gern berühren. Sie windet sich aalgleich davon und Stillhalten fällt ihr sehr schwer. Sie ist in unbekannten Situationen schnell überfordert und möchte sich entziehen bzw. zeigt Übersprungshandlungen.

\section{Untersuchung und Behandlung}

Normalerweise beginne ich mit einer Allgemeinuntersuchung und einer osteopathischen Übersichtsuntersuchung des gesamten Körpers. So lassen sich Bereiche auffinden, die eine genauere Betrachtung brauchen.
Aber bei Bella muss ich mir die entspannte Mitarbeit erst verdienen. Also ist das Ziel bei unserer ersten Begegnung, den Hund eine gute Erfahrung machen zu lassen und erste Behandlungsimpulse zu setzen bzw. begleiten zu können.

Somit ist bei der ersten Begegnung an ein Standardvorgehen nicht zu denken. Wir beginnen direkt auf der Bodenmatte mit viel Leberwurst aus der Hand der Besitzerin. Bella kann sich dabei immer nur kurz hinlegen und steht sehr schnell wieder auf. Sie mit Kommando hinzulegen, würde noch mehr Druck bedeuten. Daher suche ich die für die Hündin stressärmste Position, gehe auf maximalen Abstand und berühre sie zunächst nur mit einer Hand. Dadurch erreiche ich, dass die Hündin noch offen für die Vorgänge im Körper sein kann und die Möglichkeit hat, zumindest für kleine Momente in sich hinein zu horchen.

Ich biete Bella als ersten Kontakt meine Hand auf ihrem Sakrum an und verfolge den craniosacralen Rhythmus (CSR). Ich leite einen Stillpunkt des CSR ein. Bella knabbert hektisch an ihrem „Anker“, der Leberwursttube. Sie wendet sich aber immer wieder kurz zu mir um und schaut, was ich so tue.

Nun darf ich vorsichtig meine beiden Hände laterolateral (I/I) ans Becken legen. Ich spüre die seitliche Verbindung, den Kontakt zur LWS und zur Rute. Das Becken ist vollkommen isoliert und maximal gespannt (tight). Momentan ist es ein Bereich, der nicht zum Körper gehört und kann somit auch nicht in eine Bewegung integriert werden. Die direkte Berührung an der LWS (auch tight) und der Rute empfindet Bella als zu intensiv und sie entzieht sich. Ich biete ihr ein „Fühlen des CSR“ an dem mir nächstgelegenen Bein an. Das lässt sie für einen Moment zu.

Ich kann bei der folgenden Handanlage von dorsal an den Tuber coxae und der LWS eine beginnende UnwindingBewegung zwischen Becken und LWS kurz verfolgen.

Ich konzentriere mich auf die Verbesserung der Präsenz des Beckens mit I/I Handanlage. Das Becken wird klarer und kann etwas Spannung loslassen. Die Differenzierung zwischen Wirbelsäule, Sakrum und Becken ist als Idee wahrnehmbar.

Dabei belasse ich es zunächst. Bella hat genug Input bekommen, um damit arbeiten zu können.

Ihre Bewegung ist nun bodeneng und die Stellung der Hintergliedmaßen auch für die Besitzerin deutlich sichtbar weniger kuhhessig. 


\section{Behandlung (22.08.2017)}

Das Gangbild hat sich merklich verändert. Die Besitzerin berichtet, dass Bella auf ebenem Gelände mit der Nase unten oder beim Bergabgehen (Nase auch unten) noch hoppelt, aber ihre Hinterfüße deutlich differenzierter benutzen kann und die Hopser weniger geworden sind. Nach der letzten Behandlung war Bella sehr müde und hat viel geschlafen. Sie müsste nun bald läufig werden.

Auch heute ist an eine geordnete Untersuchung nicht zu denken.

Als ersten Kontakt nutze ich wieder das Verfolgen des CSR am Sakrum und löse einen Stillpunkt im CSR aus. Ich kann spüren, dass das Becken seinen Platz in der Wahrnehmung des Hundes hat behalten können.

Bei der heutigen Behandlung kann ich die Rute ohne Einschränkung anfassen und nutze dies für das Begleiten eines Unwindings bis zum Release in Rute und LWS. Auch die Beziehung der WS zum Becken kann ich klären. Nicht ganz bis zum Release kann ich das Diaphragma und das knöcherne Becken begleiten, bevor Bella sich entzieht.

Aber sie hat immer wieder kurze Momente, in denen ihr Blick nach innen geht und sie sich auf die entstehenden Bewegungen und die sich lösende Spannung konzentrieren kann.

Nach der Behandlung ist Bella deutlich gestreckter in der LWS und die Hintergliedmaßen sind damit nicht mehr so sehr unter den Körper gestellt.

\section{Behandlung (07.09.2017)}

Die Besitzerin erzählt, dass Bella sich morgens inzwischen hinten auch fast „richtig“ streckt. Bisher hat sie sich vorne gedehnt und mal die LWS leicht gebeugt, aber die Streckung der Hinterbeine weit nach hinten, das ist neu. Sie hat unter dem Körper eine deutlich größere Unterstützungsfläche und kann inzwischen ohne zu Hüpfen traben und sogar richtig Treppensteigen!

Heute kann ich einen tollen Erfolg erzielen: Bella lässt mich ein komplettes Unwinding mit Stillpunkt und nachfolgendem Release in Becken und in der Verbindung zur LWS bis zum Ende begleiten. Die LWS streckt und streckt und streckt sich. Es folgt ein tiefer Seufzer.

Die Rute anzufassen geht nur für eine kurze Berührung.

Bella braucht deutlich weniger Kekse zur Unterstützung, um auf dem Behandlungstisch liegen zu können.

\section{Behandlung (25.09.2017)}

Bella entwickelt mehr Ideen, wie man denn seine Füße so setzen kann und ist geschickter in ihren Bewegungen. Sie kann die Hinterbeine weiter nach hinten stellen. Die Besitzerin unterstützt ihre Hündin durch Spaziergänge mit abwechslungsreichem Boden - sie gehen viel im Wald über Wurzeln oder Moospolster etc.

Bei der Behandlung entwickeln sich heute zunächst Unwinding und Release zwischen Rute und Becken. Bei der I/I Handanlage am knöchernen Becken kann noch mal viel Spannung gehen und Bewegung entstehen. Ich darf sogar das Zwerchfell I/I behandeln. Außerdem lege ich meine Hände auf die Hüftgelenke, spüre der Verbindung zwischen meinen Händen nach und nehme die vorherrschende Spannung raus.

Wir erhöhen den Abstand zur nächsten Behandlung auf 4 Wochen.

\section{Behandlung (23.10.2017)}

Bella ist nun läufig. Die Besitzerin sagt, sie sei jetzt richtig „nett“, interessiere sich viel mehr für ihre anderen beiden Hunde und nehme mehr Sozialkontakt auf. Vom Gemüt her sei sie für ihre Verhältnisse ausgeglichen.

Heute kann ich nach dem Einstieg über den CSR/Stillpunkt am Sakrum die Rute, die Hüften und sogar das Zwerchfell mit ventrodorsaler (v/d) Handanlage behandeln. Ich taste mich an den Atlas und das Bregma heran - das genießt Bella geradezu.

BREGMA

Als Bregma wird die Stelle des Zusammentreffens der Sutura sagittalis und der Sutura coronalis am Schädel bezeichnet. Dieser Punkt steht in enger Verbindung zum intracraniellen Membransystem und die Behandlung dort hat meist eine deutlich sichtbare Entspannung und Erleichterung des Patienten zur Folge.

\section{Behandlung (22.11.2017)}

Bellas Umweltsicherheit hat sich massiv verbessert. Sie ist jetzt auch mit der Läufigkeit durch und ist geistig wieder ein Stück erwachsener geworden. Die Besitzerin sagt, dass sich Bellas Trittsicherheit jetzt sehr stabil verbessert hat und sie eine richtig gute Koordination hat.

Bella dreht die Ellbogen noch mittelgradig ein und die kuhhessige Stellung ist als geringgradig zu bezeichnen. 
Nachdem ich bei der letzten Behandlung gut den Kopf berühren konnte, konzentriere ich mich auf den CSR (Sakrum, Diaphragma, Thoraxeingang, Schultern). Das genießt Bella sehr und schmiegt sich in meine Hände. Ich kann den Atlas noch mal in ein Release begleiten und begleite ein myofasziales Release (MFR) in LWS und der Rute.

Wir erhöhen erneut den Abstand zur nächsten Behandlung.

\section{Behandlung (10.01.2018)}

Bella wird von der Besitzerin als sehr stabil bezeichnet. Das gilt gleichermaßen für das Gemüt wie auch ihre körperliche Verfassung. Bella steht hinten relativ steil, aber deutlich schöner als bisher.

Die nächste Behandlung vereinbaren wir erst für Mai.

\section{Behandlung $(24.05 .2018)$}

Bella kann die Füße viel besser setzen und darf inzwischen sogar mit auf Bergtouren in unwegsames Gelände. Dort kann sie sich sicher bewegen und auch sicher steigen. Sie steht nach wie vor ggr. kuhhessig und dreht die Ellbogen heute nur ggr. ein.

Aber: Trotz des langen Abstandes zur letzten Behandlung "gehört" alles zu Bellas Körper dazu.

Wir arbeiten am CSR am Sakrum. Ich verfolge ein MFR an Rute und LWS und behandle das Diaphragma und nach kraniosakralen Prinzipien auch das Bregma.

\section{Verlauf}

Abschließend lässt sich berichten, dass ich Bella in unregelmäßigen Abständen von mehreren Monaten behandle, wenn die Besitzerin einen Bedarf sieht. Es ist der Hündin möglich, eine eingehende Untersuchung zuzulassen und auf dem Behandlungstisch entspannt zu sein, manchmal sogar in Seitenlage. Leckerchen bekommt sie zum Einstieg auf dem Tisch, danach braucht sie keine mehr.

Abgesehen von kleineren Spannungen, die bei einem jungen, schnellen Hund immer wieder zu finden sind, hat sich Bellas Bewegungsmuster gut stabilisiert. Sie wird immer (noch) geschickter in ihren Bewegungsabläufen und kann, mit begleitender osteopathischer Nachbalancierung, hoffentlich ohne größere Probleme, ein ausgelastetes Hundeleben führen.

\section{Mein Fazit}

Mit ausreichender Flexibilität in meiner Vorgehensweise und Achtung vor den Bedürfnissen der Patientin konnte sich diese sehr agile und impulsive Hündin mit der Zeit voll auf die Behandlung einlassen. Auch wenn ich gerade bei den ersten Begegnungen weit von dem entfernt war, was ich mir so vorgestellt hätte, hat der Körper sich genau das geholt, was ihm weitergeholfen hat.

Grundsätzlich empfinde ich es als sehr hilfreich, einem Untersuchungsschema zu folgen. Dabei erhebe ich von kaudal nach kranial Befunde im kraniosakralen, parietalen und viszeralen System. So kann ich feststellen, welche Bereiche in unphysiologischen Spannungszuständen sind, wo ich genauer noch untersuchen muss und welches System bzw. an welcher Stelle ich behandeln sollte.

Mir erleichtert ein für mich festgelegter Untersuchungsablauf die genaue Befunderhebung und die geforderte Dokumentation von Diagnosen und Behandlungen.

Der vorliegende Fall zeigt aber deutlich, dass manche Patienten eine komplette Untersuchung nicht von Anfang an erlauben und zunächst einen anderen Zugang für eine erfolgreiche Behandlung benötigen.

Autorin

\section{Anne Lisa Louis}

Studium der Tiermedizin in Hannover, niedergelassen (Schwerpunkt Osteopathie) seit 2017; div. Fortbildungen Osteopathie, Homöopathie u. Verhaltenstherapie Mitgliedschaften: TAPO (Tierärztlicher Arbeitskreis für Physiotherapie und Osteopathie), GGTM, ATF

\section{Korrespondenzadresse}

\section{Dr. med. vet. Anne Lisa Louis}

Point 2

83246 Unterwössen

info@tierosteopathie-chiemgau.de www.tierosteopathie-chiemgau.de

\section{Literatur}

[1] Könneker H Reiter U. Osteopathie in der Kleintierpraxis. 1. Aufl. Stuttgart: Sonntag-Verlag; 2010

[2] Löwe RU. Craniosacrale Heilkunst. 5. Aufl. Bielefeld: Aurum; 2017

[3] Milne H. Aus der Mitte des Herzens lauschen Bd. 1. 2. Aufl. Petersberg: Via Nova; 2007

[4] Nickel R Schummer A Seiferle E. Lehrbuch der Anatomie der Haustiere Bd 1. 8. Aufl. Stuttgart: Parey/Enke; 2003

[5] Upledger JE Vredevoogd JD. Lehrbuch der Craniosacralen Therapie I. 7. Aufl. Stuttgart: Karl F. Haug-Verlag; 2016

\section{Bibliografie}

DOI https://doi.org/10.1055/a-1000-1062 Zeitschrift für Ganzheitliche Tiermedizin 2020; 34: 65-68 (c) Georg Thieme Verlag KG Stuttgart · New York ISSN 0939-7868 